
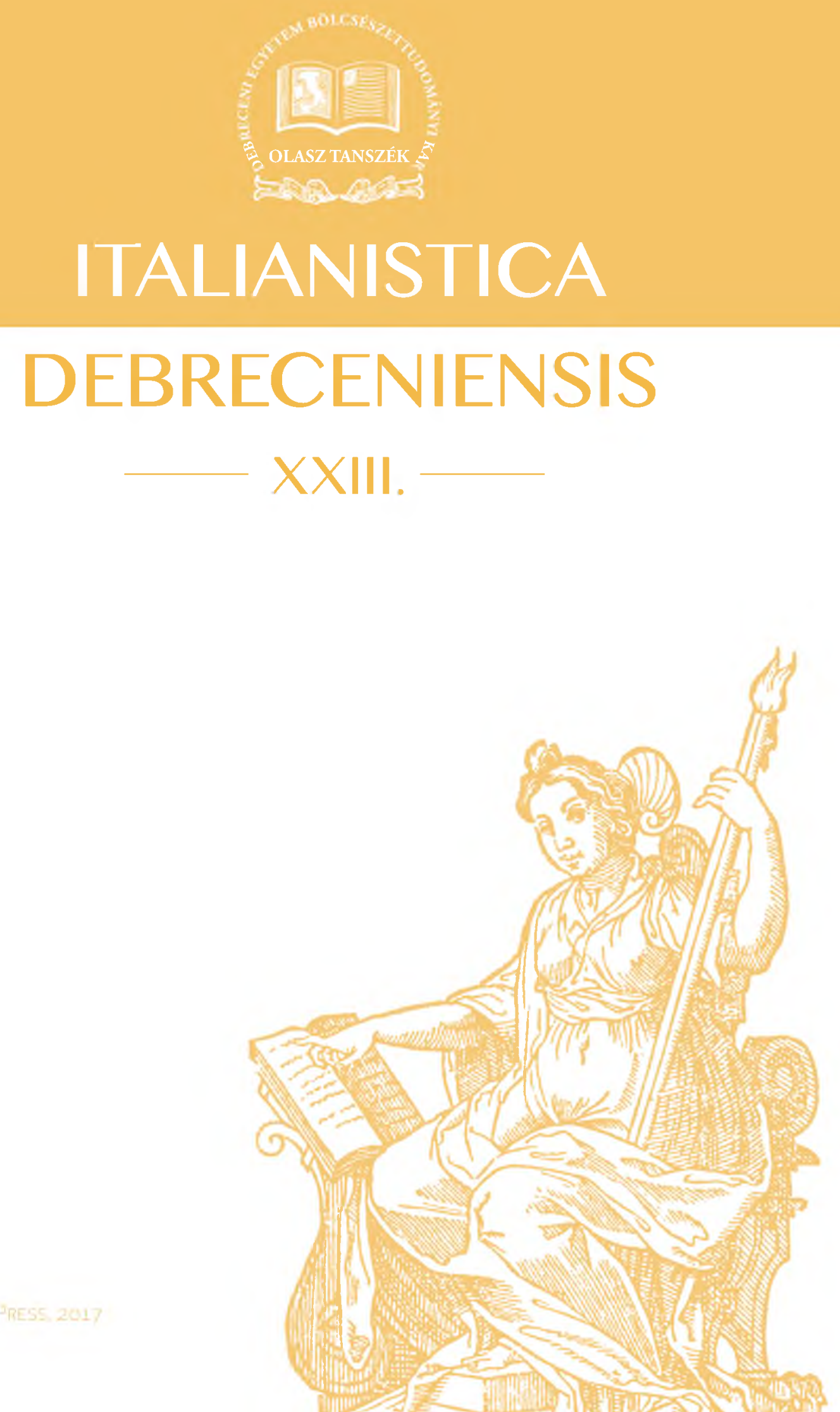


\section{ITALIANISTICA \\ DEBRECENIENSIS XXIII.}

rivista ufficiale del Dipartimento di Italianistica dell'Università di Debrecen 


\title{
Italianistica Debreceniensis
}

rivista ufficiale del Dipartimento di Italianistica dell’Università di Debrecen official journal of the Italian Studies Department of the University of Debrecen

\author{
Direttori: \\ László Pete, Paolo Orrù
}

\section{Comitato redazionale / Editorial Board:}

Barbara Blaskó, Zsigmond Lakó, Imre Madarász, István Puskás, Orsolya Száraz

\author{
Comitato scientifico / Committee: \\ Andrea Carteny (Università degli Studi di Roma "La Sapienza") \\ Vera Gheno (Università degli Studi di Firenze/Accademia della Crusca) \\ Andrea Manganaro (Università di Catania) \\ Elena Pirvu (Universitatea din Craiova) \\ Dagmar Reichardt (Latvijas Kultūras Akadēmija) \\ Péter Sárközy (Università degli Studi di Roma "La Sapienza") \\ Antonio Sciacovelli (Turun yliopisto) \\ Maurizio Trifone (Università degli Studi di Cagliari) \\ Ineke Vedder (Universiteit van Amsterdam) \\ Franco Zangrilli (The City University of New York)
}

Italianistica Debreceniensis is a peer-reviewed journal. It appears yearly and publishes articles and reviews in Italian and English. Articles submitted for publication in the journal should be sent by e-mail attachment (as a Word document) to one of the Editors: Paolo Orrù

(paolo.orru@arts.unideb.hu), László Pete (pete.laszlo@arts.unideb.hu).

Italianistica Debreceniensis si avvale della valutazione peer-review. Ha cadenza annuale e pubblica articoli in Italiano e Inglese. Le proposte di contributo per la pubblicazione possono essere inviate per e-mail (in un file Word) a uno dei due direttori: Paolo Orrù (paolo.orru@arts.unideb.hu), László Pete (pete.laszlo@arts.unideb.hu).

Books for review should be sent at the following address /

I libri da recensire possono essere spediti all'indirizzo:

Debreceni Egyetem, Olasz Tanszék, 4002 Debrecen, Pf. 400.

For more information visit our website: http://italdeb.arts.unideb.hu/index.php/italdeb 


\section{Indice}

\section{Articoli - Articles}

Tancredi Artico: Danese Cataneo, «felicissimo spirito» nelle carte tassiane.

L’Amor di Marfisa e la Gerusalemme liberata 8

Adele Bardazzi: «Occasioni» e «moments of being»: il modernismo di Montale

Julia Dabasi: Il legame tra lo spazio e l'individuo in Petrarca e Leopardi 38

Elisa Della Mea: Marano: una fortezza contesa. La crisi dei rapporti politicodiplomatici tra le principali potenze europee a seguito del colpo di mano su Marano del 1542 46

Marco Giani: «Donna, che fosti tra le donne un Sole»: sui tentativi poetici giovanili di Paolo Paruta (metà XVI sec.) 60

Eleonora Mamusa: The exaltation of Italian national identity in Matteo Renzi's discourse 74

NoÉмт Óтотт: «Siete voi qui, ser Brunetto?». I volti di Brunetto Latini: rappresentazione e autorappresentazione 96

Diego Stefanelli: Appunti sulla stilistica (italiana) di László Gáldi 108

FRAnCo Zangrilli: Max Gobbo e la riscrittura fantastica di un periodo rinascimentale 122

\section{Recensioni - Book reviews}

Dagmar Reichardt e Carmela D'Angelo, Moda made in Italy. Il linguaggio della moda e del costume italiano, Firenze, Franco Cesati, 2016 (Luigi Saitta) ......... 132

Franco Zangrilli, Il piacere di raccontare. Pavese dentro il fantastico postmoderno, Palermo, Dario Flaccovio Editore, 2017 (Biagio Coco) 


\title{
Marano: una fortezza contesa. La crisi dei rapporti politico-diplomatici tra le principali potenze europee a seguito del colpo di mano su Marano del $1542^{1}$
}

\author{
di Elisa Della Mea
}

\begin{abstract}
Venice's reconquest of Marano in 1542 was a key moment in the history of the Republic. The fortress of Marano was in fact at the top of its glory between the XV and XVI century, when it was contested between Austria and Venice. When it fell in the hands of Austria in 1513, Venice tried to reconquest it with every possible means. After years of unsuccessful attempts, the feat was carried out by Beltrame Sacchia, an ambitious and adventurous merchant from Udine, who occupied the fortress in 1542 in name of the King of France. This article analyses the repercussions of Marano's reconquest on European political equilibrium. What happened on the morning of January 2, 1542, as well as making a turning point in the boundary dynamics between Venice and the Austrian, deeply damaged the diplomatic relations between the main powers of Europe: the Venetian Republic, France, the Empire and the Ottomans.
\end{abstract}

\section{Introduzione}

Dopo la guerra della Lega di Cambrai, per la Serenissima si fece sentire con sempre più insistenza il problema del rinnovamento complessivo delle strutture difensive dei suoi territori di Terraferma, affrontato soprattutto in due periodi principali: quello emergenziale delle Guerre d'Italia, di scarsa adeguatezza di fortezze, presidi e artiglierie/munizioni e di spauracchi per gli eserciti stranieri; e il mezzo secolo 1540 circa-1593, caratterizzato dalla progressiva rifortificazione della Terraferma grazie a investimenti finanziari fluttuanti ma consistenti. ${ }^{2}$ Il quadro dell'assetto difensivo che risultava dalla relazione dei Sindici Inquisitori del 1543 non era affatto entusiasmante: nei presidi erano numerose le mancanze,

\footnotetext{
${ }^{1}$ Il presente articolo è una rielaborazione di parte della tesi di Dottorato di Ricerca in Storia: Culture e Strutture delle Aree di Frontiera (ciclo XXVII), dal titolo La sicurezza incerta del confine orientale. Venezia, Friuli e Istria dalle guerre d'Italia al progetto di Palmanova (c. 1494-1593), discussa il 26 maggio 2016 presso l'Università degli Studi di Udine. Nelle note si useranno le seguenti abbreviazioni: ASV: Archivio di Stato di Venezia; PSCC: Provveditori Sopraintendenti alla Camera dei Confini.

${ }^{2}$ Cfr. M. E. Mallett, J. R. Hale, The military Organization of a Renaissance State. Venice c. 1400 to 1617, Cambridge, Cambridge University Press 1983; E. Concina, La macchina territoriale. La progettazione della difesa nel Cinquecento veneto, Bari, Laterza, 1983.
} 
frequenti le indiscipline, e mal governate le vettovaglie e munizioni, ed era ancora preoccupante l'inadeguatezza strutturale di mura e piazzeforti rispetto alle nuove tecnologie militari.

Se fra secondo e terzo decennio del '500 le lotte per l'egemonia in Italia continuarono ad essere combattute soprattutto nella pianura lombarda, costringendo Venezia a privilegiare la parte centrale e quella occidentale dello stato di Terraferma per gli interventi sulle strutture difensive (Treviso, Padova, Verona, Brescia, Crema e le fortificazioni di Asolo, Legnago e Peschiera), attorno al 1525, invece, a due anni dall'ascesa di Andrea Gritti al dogado, vennero proposti ulteriori interventi, compresa la fortificazione di Udine e Venzone a sbarramento delle vie che conducevano in Germania attraverso la Carnia e il Canal del Ferro.

Quando verso la fine del 1531 si profilò all'orizzonte il pericolo di uno scontro con l'impero ottomano, la dimensione del problema del rinnovamento complessivo delle strutture difensive dei territori dipendenti da Venezia si allargava ulteriormente a comprendere i distretti nord-orientali della Terraferma e l'area adriatica. A questo scopo, nella primavera del 1532, il duca d'Urbino Francesco Maria della Rovere raggiungeva Venezia, sulla spinta del clima di emergenza e probabilmente con l'appoggio della cerchia del Gritti, per fronteggiare la questione delle nuove fortificazioni.

Per il Friuli il problema nodale era costituito dal recupero e dalla fortificazione del centro portuale di Marano, allora in possesso degli arciducali: progetto che il duca d'Urbino suggeriva di attuare con ogni mezzo in quanto Marano valeva, come base per le operazioni navali nell'alto Adriatico, molto più di Trieste. Una volta provveduto a questo, «con quatro luochi, Osoppo, Udine, Sacil et Marano si faceva una buona colleganza per la fortezza de Venezia», ${ }^{3}$ costituendo così un ulteriore sistema territoriale di fortificazioni, strettamente correlato a quello già propriamente veneto. Il duca ricordava che Marano era potenzialmente uno dei «più importanti luoghi, havendola vostra Serenità in suo potere, per diffesa del Friuli et resto del Stato di vostra sublimità et non havendolo delli più atti ad offender questa città di Venezia». ${ }^{4}$ Perciò il duca incitava la Repubblica a «procurar in ogni modo di riguadagnar Marano, il che reputava che potesse esser men difficile a questi tempi, che ad altri, ritrovandosi come si trovavano queste guerre in piedi, et il re dei Romani nella necessità del denaro». ${ }^{5}$ Il consiglio, però, era di muoversi cautamente nelle operazioni di recupero della fortezza, facendo credere agli arciducali di voler conquistare anche altri luoghi, per confonderli «acciò non si

\footnotetext{
${ }^{3}$ E. Concina, La macchina territoriale, cit., p. 29.

${ }^{4}$ Ivi, p. 33.

${ }^{5}$ ASV, PSCC, b. 169, reg. VI (Friuli), cc. 1-5, 17 settembre 1532.
} 
scoprisse l'importantia di questo et il conto che se ne tiene, che causeria tanto più difficoltà a conseguirlo» ${ }^{6}$.

\section{Importanza strategica di Marano}

Voluta dal patriarca Popone nel XI secolo come la più importante difesa del patriarcato dalla parte del mare, Marano, che allora era solo una piccola villa di Aquileia, conobbe tra XV e XVI secolo (prima dell'ideazione della fortezza di Palmanova) il suo periodo di maggior splendore. Elevatasi al rango di comunità, venne dotata, come Aquileia, di un podestà e nel Quattrocento subì delle generali opere di fortificazione, attraverso l'erezione di terrapieni e la costruzione di molini e saline. ${ }^{7}$ Contesa da Venezia e dagli arciducali, la fortezza era un'imprescindibile difesa, posta tra l'Istria e la laguna veneziana, a garanzia anche del traffico mercantile in quel tratto di Adriatico, a cominciare dal porto di Lignano, parte integrante della piazzaforte maranese. Girolamo di Porcia, infatti, descrisse Marano come «luogo fortissimo di muraglia, terrapieni, fosse larghissime dove entra la marina, e si può girare in una galea; ha un porto bellissimo, e capace di più di 400 velle, detto Lignano». ${ }^{8}$ Anche dalla scrittura presentata a Venezia nel 1532 da Giacomo Leonardi, segretario del duca d'Urbino, emergeva in modo inequivocabile la vocazione di Marano a baluardo della difesa del Friuli e punto di collegamento della Dominante con il resto dei suoi territori più orientali. Forte della sua vantaggiosa posizione, Marano «trovandosi in mani altrui pregiudicava altamente gli interessi della Repubblica, tanto dal lato strategico quanto da quello economico» ${ }^{9}$. In caso di guerra, poiché priva di una più facile comunicazione con il Friuli (la cosiddetta "Strada di Levada" passante attraverso le paludi, che avrebbe dovuto garantire alla fortezza il rifornimento delle derrate alimentari e i traffici verso l'entroterra veneto, venne costruita solo tra il 1611 e il 1612), la fortezza «sarebbe potuta venire in grave distretta» ${ }^{10}$ nel momento in cui gli arciducali avessero ricevuto aiuti dalle navi spagnole attraccate a Lignano. In tempo di pace, invece, questo porto, non accessibile alle imbarcazioni venete, avrebbe consentito agli arciducali di trasportare in Friuli olio, sale e granaglie senza pagare i dazi d'importazione alla Repubblica. ${ }^{11}$

\footnotetext{
${ }^{6}$ Ibid.

${ }^{7}$ Per un approfondimento più puntuale della storia di Marano, cfr. R. Olivotto, Marano lagunare, volo attraverso i secoli fino al giorno dell 'inaugurazione dell'Acquedotto, Cividale, Tipografia Fulvio Giovanni, 1892.

${ }^{8}$ G. (di) Porcia, Descrizione della Patria del Friuli, Udine, Tipografia del patronato, 1897, pp. 75-76.

${ }^{9}$ A. Puschi, Attinenze tra Casa d'Austria e la Repubblica di Venezia dal 1529 al 1616, Trieste, Tipografia del Lloyd austro-ungarico, 1879, p. 7.

${ }^{10} \mathrm{Ibid}$.

${ }^{11}$ Il luogotenente Andrea Foscolo nel 1525 definì la fortezza di Marano «de grandissimo danno a li dacii di vostra
} 
Questa fortezza, affacciata sulla laguna, aveva un'invidiabile valenza strategica, il cui possesso, vista la situazione critica dei primi decenni del Cinquecento, avrebbe garantito ai veneziani di «conservar lo stado de questa Patria». Da Marano si potevano, infatti, controllare le foci del Tagliamento e del fiume Ausa e il traffico sulle strade retrostanti; senza questo baluardo, il controllo veneziano dei porti imperiali di Trieste e $\mathrm{S}$. Giovanni di Duino sarebbe stato di gran lunga più difficoltoso. La sua occupazione era determinante quindi per la sicurezza stessa del governo lagunare e andava salvaguardata a qualsiasi costo. Sfortunatamente per i veneziani, però, Marano, sotto il loro controllo sin dal 1420, finì in mano arciducale nel 1513, nel periodo turbolento delle Guerre d'Italia. Da quel momento fino al 1542, la Repubblica non lasciò intentato nessun mezzo per riappropriarsi della fortezza: numerosi furono i tentativi (tutti documentati fra il 1515 e il 1529) messi in atto per scalzare gli arciducali, nella certezza da parte veneta che «tuti li Maranesi sono satii de stare soto il dominio barbaricho». ${ }^{12}$

\section{La presa di Marano del 1542 e il gioco delle diplomazie}

\subsection{La genesi dell'impresa}

Il mercante udinese Beltrame Sacchia fu la pedina utilizzata da Venezia per portare a compimento la presa della fortezza di Marano, dopo anni di tentativi andati a vuoto. Che ci fosse la Repubblica dietro questa ben architettata impresa è ora un dato certo e incontrovertibile, suffragato da numerose prove e indizi, anche se all'epoca il governo lagunare si dimostrò inizialmente estraneo ai fatti, per mantenere rapporti di "buon vicinato" con l'arciduca Ferdinando. La trama che sta dietro alla presa di Marano è fittamente intrecciata e vede come principali protagonisti la Repubblica e i francesi, nelle figure rispettivamente degli aderenti al "partito filofrancese" da una parte e dell'ambasciatore francese a Venezia Guillaume de Pellicier, vescovo di Montpellier, dall’altra.

La genesi dell’impresa è rintracciabile nella nomina del Sacchia a cavaliere nobile, concessagli dal re di Francia Francesco I nel marzo del $1541 .^{13}$ Beltrame (1507-1550) era figlio del mercante udinese Lorenzo Sacchia, uno degli uomini d'affari più facoltosi del capoluogo friulano, nonché uno dei rappresentanti poli-

\footnotetext{
sublimità, per el condur de le merchantie prohibite: et similiter perché de Istria se trazeno oglii de Pugia soto pretesto che siano istriani», Relazioni dei rettori veneti in Terraferma, I, La Patria del Friuli (Luogotenenza di Udine), a cura dell'Istituto di storia economica dell’Università di Trieste, Milano, Giuffrè, 1976, p. 4.

${ }^{12}$ ASV, Capi Consiglio dei Dieci, Lettere di rettori, b. 188, f. 18, 26 febbraio 1516. Sui vari tentativi di conquista veneta dopo il 1513, si rimanda a E. Della Mea, Beltrame Sacchia e la riconquista di Marano (1542-1550), «"Ce Fastu?", Rivista della Società Filologica Friulana», LXXXVIII (2), 2012, pp. 213-239.

${ }^{13} \mathrm{G}$. Cogo, Beltrame Sachia e la sottomissione di Marano al dominio della Repubblica veneta (con nuovi documenti), in «Nuovo Archivio Veneto», XIV/I, 1897, pp. 32-33.
} 
tici della clientela popolare e filosavorgnana udinese di inizio Cinquecento. ${ }^{14} \mathrm{La}$ carriera mercantile di Beltrame fu in continua ascesa, favorita sia dai contatti del padre sia dalle sue capacità imprenditoriali: negli anni riuscì a incrementare il giro d'affari della famiglia prima importando olio e granaglie dall'Istria e dalla Carniola, poi assumendo gli appalti dei dazi del vino e del sale nella Patria e, infine, occupandosi del trasporto di ingenti quantità di grano a Venezia dall'Ungheria (1540-41). La sua smisurata ambizione personale, unita al desiderio di conquistarsi un posto più elevato in società, per scrollarsi di dosso i limiti impostigli dalla sua condizione di popolano udinese, lo spinsero ad assumere atteggiamenti e stili di vita ben al di sopra delle sue possibilità. Questo, gli permise anche di stringere utili contatti con gli ambienti politici veneziani. Da qui probabilmente ebbe origine l'idea di mettersi al servizio del governo lagunare organizzando la riconquista della fortezza maranese. L'ipotesi regge, dal momento che lo stesso Sacchia dirà al bailo di Costantinopoli che «la cosa di Maran l'ho fatta non per farli despiacer [a Venezia], anzi io me offersi per inanti di far per voi quel che ho fatto per il Re». ${ }^{15}$ Questo significa che il Sacchia aveva esposto la sua idea a Venezia ben prima di ricorrere alla corte del re di Francia e che, in ogni caso, Venezia non era del tutto estranea alla faccenda come poi volle far credere.

In un documento del 12 gennaio 1542, infatti, alcuni giorni dopo il colpo di mano, il Senato ordinò al luogotenente di rintracciare il Sacchia per suggerirgli di «tenir questo loco nelle mani sue ad instantia nostra, et volendo noi che questa pratica passi secretissima, acciò che se habbi bona causa de mandar uno ad esso Beltrame senza dar suspitione ad alcuno». ${ }^{16}$ L'intento era di fargli sapere che, se avesse tenuto Marano a nome e per conto di Venezia, l'avrebbero nominato conte concedendogli una provvigione di 1.000 ducati annui. ${ }^{17}$

In realtà però la collaborazione non andò in porto e il Sacchia preferì recarsi in Francia, probabilmente su consiglio dell'ambasciatore francese a Venezia Guillaume de Pellicier, che nella città lagunare era molto conosciuto e poteva contare su amicizie influenti. Dal 1539 il diplomatico francese, infatti, si applicò con successo all'attuazione dei piani politici del suo sovrano volti a ostacolare la politica di Carlo V in Italia, a staccare Venezia dalla Lega che aveva concluso con il papa e l'imperatore, a riconciliarla con la Sublime Porta ottomana, alleata della Francia,

\footnotetext{
${ }^{14}$ L. e G. Amaseo, Diarii udinesi dall'anno 1508 al 1541, Venezia, 1884, p. 26. Lorenzo Sacchia era considerato dai suoi contemporanei uno dei dieci uomini più influenti di Udine («quando talhora si nominavano otto, overo diece di questa città, il detto messer Lorenzo era nominato fra quelli»). Per ogni ulteriore approfondimento sulle vicende familiari dei Sacchia padre e figlio mi permetto di rimandare a Della Mea, Beltrame Sacchia.

${ }^{15}$ E. Della Mea, Beltrame Sacchia e la riconquista di Marano (1542-1550), tesi di laurea specialistica in Storia e civiltà europee, Università degli Studi di Udine, Facoltà di Lettere e Filosofia (a.a. 2009-2010), p. 97.

${ }^{16}$ Ivi, p. 95.

${ }^{17} \mathrm{Ibid}$.
} 
e a spingerla a unirsi alla Francia stessa. Il Pellicier a tal fine si servì di una rete spionistica, i cui agenti si reclutavano nella nobiltà veneziana e tra gli stessi alti funzionari delle magistrature venete. ${ }^{18}$

\subsection{L'impresa, i suoi protagonisti e le prime reazioni}

Sulle gesta compiute dal Sacchia in quel fatidico mattino del 2 gennaio $1542 \mathrm{~mol}-$ to è stato scritto, soprattutto nelle cronache del tempo. ${ }^{19} \mathrm{Ci}$ limiteremo a dire che fu lui stesso a scegliere i tempi e i modi dell'azione, compiuta di sorpresa, con due barche cariche di grano, in cui egli, novello Ulisse, aveva fatto nascondere una sessantina di uomini armati. Una volta giunto in prossimità della fortezza, al Sacchia, «coperto d'una peliza lunga con la spada sotto et un brochiero di ferro», venne chiesto

quello portava in esse barche, disse "To ho un poco di formento. Io voleva andar a Venetia con esso, ma m'dato il vento contrario et per esser poco, voglio sbarcarlo qui per comodita et beneficio della terra" [...]. Smontato il Sacchia in terra visto il comodo, disse con horibil voce "Fuora formento". Caziò mano alla spada, gettato la peliza, gittato le stuore in aqua, li soldati saltati fora, pigliar la porta ferendo et amazando quanti trovava per strada, dove li poveri maranesi fugiva a scondersi como conigli, [...] onde in pocho d'hora presero la terra et il capitano Herman Gruennoffer nel palazo et lo posero in pregione, et serato la porta di terra et di mare cridando "Marcho, Marcho, Franza, Franza, Turcho, Turcho". ${ }^{20}$

La presa di Marano ebbe un'immediata ripercussione sui fragili equilibri europei e, da piccola scaramuccia di confine, si trasformò in un incidente diplomatico che rischiò seriamente di compromettere i rapporti tra la Repubblica e gli arciducali. Venezia, nei giorni immediatamente successivi al fatto, si mosse con cautela per preservare la sua "presunta" neutralità sulla faccenda («essendo la intention nostra de star neutrali et non ne impedir circa le cose de Marano a favore né de l'una né de l'altra parte»). Per questo, con una lettera del 23 gennaio, il Consiglio dei Dieci ingiunse al capitano di Capodistria di far «intender alli prefati Camerlengo et ser Pietro Marcello [che erano andati in Friuli per aiutare il Sacchia, assieme ad

\footnotetext{
${ }^{18}$ G. Gullino, Marco Foscari (1477-1551). L'attività politica e diplomatica tra Venezia, Roma e Firenze, Milano, Franco Angeli, 2000, con relativa bibliografia.

${ }^{19}$ ASV, PSCC, b. 218, "Relatione dela presa di Marano 1542" di Durastante Leoncino da Ontognano; M. Guazzo, Historie di tutti i fatti degni di memoria nel mondo successi del 1524 fino a l'anno 1549, Venezia 1569, pp. 288r-290r.

${ }^{20}$ ASV, PSCC, b. 218, "Relatione dela presa di Marano 1542" di Durastante Leoncino da Ontognano, ff. 4r-4v.
} 
altri 25 uomini] che immediate se levino da Marano con tutti» e tornino a Capodistria. ${ }^{21}$ Negli stessi giorni, il Senato scriveva al luogotenente Contarini complimentandosi per la sua decisione di contattare gli agenti di Ferdinando a Gradisca, «dechiarandoli come ciò sia seguito senza colpa nostra», sottolineando ancora una volta come questo fatto fosse stato causato da sudditi veneti senza però «alcuna nostra saputa».22

L’atteggiamento "troppo" neutrale di Venezia, però, non poteva non insospettire i ministri spagnoli della corte di Ferdinando. L’ambasciatore veneto in Francia, infatti, riferiva che l'ambasciatore cesareo «è pieno di molto sospetto dell'intelligentia che la celsitudine vostra habbia havuta in questo furto di Marano», nonostante i suoi tentativi di «levarglielo in tutto».23

Quello che accadde la mattina del 2 gennaio 1542, oltre a segnare un punto di svolta nelle dinamiche confinarie tra Venezia e gli arciducali, mise fortemente in crisi i rapporti diplomatici tra le principali potenze europee: la Repubblica di Venezia, la Francia, l'Impero e la Sublime Porta ottomana.

\subsection{Marano e la Francia}

La notizia della "derobatione" di Marano arrivò alla corte di Francia solo 20 giorni dopo. La presa di posizione di Francesco I di fronte a quanto accaduto è netta e decisa: «ella non la volea [Marano], ponto». All'ambasciatore veneto riferì che, vista la «bona et ferma intentione di mantenere l'amicitia et parentella con l'imperatore et con Fernando suo fratello, cioè re de Romani», non voleva in nessun modo accettare la fortezza. ${ }^{24}$

Secondo il sovrano, la fortezza era stata occupata da alcuni «francesi banditi», accusati di «crimini pessimi», che avrebbero compiuto l'impresa solo per tornare nelle sue grazie; non ottenendo «cotal gratia» Francesco era sicuro che avrebbero tentato di vendere Marano al miglior offerente: Ferdinando d'Asburgo o, peggio, «al Turco, ricordandone lo attrovarsi fuora di Barbarossa con quella potente armata chel si attrova». I giorni seguenti furono molto concitati alla corte di Francia. L'ambasciatore veneziano riferiva al governo lagunare come molti della corte sospettassero di un coinvolgimento della Serenissima nell'impresa («la saperà esser opinione in molti di questa corte che in questa espeditione di Marano la Serenità vostra gli habbia havuta mano»). Perfino il nunzio pontificio ebbe di che obiettare in merito all'estraneità del sovrano francese, poiché «era publica

\footnotetext{
${ }^{21}$ ASV, Consiglio dei Dieci, Secreta, reg. V, 23 gennaio 1542.

${ }^{22}$ ASV, Deliberazioni Senato, Secreta, reg. 61, 12 gennaio 1542.

${ }^{23}$ ASV, Archivi propri ambasciatori, Francia, b. 1, 6 febbraio 1542.

${ }^{24}$ Ivi, 22 gennaio 1542.
} 
voce per Italia che lei havesse ordinata tale impresa». ${ }^{25}$ Francesco I difese vigorosamente la sua posizione, anche nei mesi seguenti. In marzo, alla presenza dei capi del Consiglio dei Dieci, il segretario dell'ambasciatore francese Guillaume Pellicier ribadì che «el re christianissimo non vol Maran» ${ }^{26}$ Lo stesso Pellicier, alcuni giorni dopo, confermò che il sovrano «non ha voluto quello loco»; Marano non era di nessun interesse per Francesco I in quanto era troppo lontano dai suoi possedimenti francesi. ${ }^{27}$

È improbabile che il re di Francia non avesse saputo nulla riguardo all'impresa di Marano, anche considerando il ruolo svolto dal suo ambasciatore a Venezia nel reclutare il Sacchia e nel sovvenzionare con uomini e denaro il progetto di conquista. Beltrame, infatti, secondo quanto riferito da Francesco I al consigliere dell'imperatore Granvelle, «dice ad ognuno pubblicamente che tutto ciò chel ha fatto di esso Marano, lo ha fatto ad instantia di questa maestà con intelligentia et ordine dello ambasciatore suo, lamentandossi di non ne ricever anchora alcuna remuneratione». ${ }^{28}$ Ma il sovrano continuava ad insistere di non volere la fortezza per sé e di non aver mai ordinato a costui di conquistarla. Anzi, l'anno seguente, alla presenza dell'ambasciatore veneto, mostrò di non conoscerlo «né per Sachia, né per Beltrame» e alla replica «Quello di Maran, Sire», si lasciò scappare in francese l'esclamazione «le fol colui, che vol dir "mato" in lingua italiana. Et soggionse 'costui è cattivo homo, voleva che io lo investisse di Marano, et li desse mandato che l'andasse al signor turco et levasse 10 galee da tenir in quel porto, con le qual faceva chimere contra Venetia'». ${ }^{29}$ L'atteggiamento di Francesco I si spiega considerando la particolare congiuntura politico-militare che si era creata dopo l'incidente diplomatico del luglio 1541 (l'assassinio di un agente del francese a Milano), che aveva pericolosamente incrinato i rapporti franco-imperiali. Non era ancora una guerra dichiarata, ma i fatti di Marano potevano farla scoppiare. E, probabilmente, questo non era il momento giusto.

Dopo l'acquisto veneziano della fortezza nel 1543, però, sarà molto più chiaro perché il sovrano francese avesse scelto di negare il suo coinvolgimento nell'impresa del Sacchia. Da un colloquio tra Bernardo Navagero, ambasciatore veneto presso la corte di Carlo V, e il diplomatico francese, infatti, quest'ultimo rivelò che Marano era stato per Francesco I uno strumento utile per "tastare il polso" di

\footnotetext{
${ }^{25}$ Ivi, 24 gennaio 1542.

${ }^{26}$ ASV, PSCC, b. 214, 10 marzo 1542.

${ }^{27}$ Ivi, 15 marzo 1542. L'anno seguente il Pellicier, ormai destituito dall'incarico di ambasciatore a Venezia dopo lo scandalo del complotto spionistico, riconfermò che i fatti di Marano erano accaduti all'insaputa del re di Francia.

${ }^{28}$ ASV, Archivi propri ambasciatori, Francia, b. 1, 13 marzo 1542.

${ }^{29}$ ASV, PSCC, b. 214, 8 giugno 1543.
} 
sua maestà cesarea e saggiarne le reazioni in vista di un futuro conflitto. ${ }^{30}$ Francesco I, in realtà, voleva dare la fortezza alla Serenissima («vi ho ditto sempre che Maran lo tengo per la Signoria»), come ebbe modo di assicurare monsignor Armiraglio all'ambasciatore veneziano, escludendo fermamente che questa potesse essere ceduta ai Turchi.

\subsection{Marano e l'Impero Ottomano}

Il clima di incertezza che si era creato attorno al possesso di Marano, secondo Francesco I, avrebbe potuto determinare il passaggio della fortezza «nelle mani del Turco», e questo per le ambascerie europee, soprattutto per quella arciducale, avrebbe potuto rappresentare un grave problema. ${ }^{31}$ In Ungheria, infatti, dall'agosto del 1541 l'esercito di Solimano occupava Ofen (Buda) e minacciava gli altri domini ereditari degli Asburgo, e nel Mediterraneo i Turchi si accingevano a passare all'offensiva, dopo il fallimento dell'impresa tentata in quell'ottobre da Carlo V ad Algeri. L'ambasciatore Marino Giustinian scriveva da Vienna che, se malauguratamente anche la Germania fosse passata ai Turchi, «gli stati di vostra Serenità sariano i primi assaliti [...] e giudicherei che a quella ruina non vi fosse riparo». Lo stesso nunzio pontificio, all'indomani del colpo di mano del Sacchia, esprimeva la sua preoccupazione al riguardo, persuadendo l'ambasciatore veneziano affinché riferisse al Consiglio che «le excellentie vostre non ne debbano lasciare tratto per farsi capitare esso Marano nelle mani, perché oltra che non può star nelle miglior mani, essendo già stato suo et così vicino le el liberarebbeno dil pericolo di andare nelle mano del Turco». ${ }^{32}$

Marano all'improvviso si trovava catapultata al centro degli interessi politici delle maggiori potenze dell'epoca, punto focale all'interno del grande conflitto europeo che contrapponeva Carlo V e il fratello contro Francesco I alleato di Solimano il Magnifico. In tutto questo, Venezia inizialmente preferì mantenersi neutrale e «lasciarli fare a loro, non volendo impacciarsi più per l'uno che per l'altro», preferendo aspettare il momento più opportuno per esporsi in maniera diretta. Come poi fece l'anno seguente, quando, alla luce dell'alleanza tra la Francia e la Sublime Porta, dichiarò che se non fosse prontamente intervenuta, Pietro Strozzi, minacciato da crescenti forze imperiali, avrebbe ceduto Marano al sultano turco..$^{33}$

Nella primavera del 1542, all'ambasciatore ottomano Yunus Bey, che era arrivato a Venezia per assistere al giuramento di pace che la Repubblica aveva con-

\footnotetext{
${ }^{30}$ ASV, Archivi propri ambasciatori, Germania, b. 1, 25 gennaio 1544.

${ }^{31}$ ASV, Archivi propri ambasciatori, Francia, b. 1, 24 gennaio 1542.

${ }^{32}$ Relazioni di ambasciatori veneti al Senato, Germania (1506-1554), vol. II, a cura di L. Firpo, Torino, Bottega d'Erasmo, 1970, p. 383, 25 gennaio 1542.

${ }^{33}$ ASV, Archivi propri ambasciatori, Francia, b. 1, 13 maggio 1542.
} 
cluso con l'impero ottomano nel 1540, era stato affidato da Solimano un compito delicato: avrebbe dovuto sollecitare i Veneziani ad appoggiare la Francia, assicurandosi che la Repubblica inviasse viveri alle truppe francesi che si trovavano in precaria situazione a Marano e non si mostrasse ben disposta verso Carlo V. ${ }^{34}$ Non stupisce la fredda reazione dell'ambasciatore turco («esso Yanusbey si era diportato molto freddamente») di fronte alla decisione del Senato veneziano di «voler star neutrale». Questo costrinse Solimano, alcuni mesi dopo, a richiamare il doge, affinchè rispettasse la promessa di lasciare che gli uomini del sultano «vadino et tornino per condur vittualia alla fortezza nominata Maran, che il prenominato [il re di Francia] tolse a Ferdinando». La condizione di pace tra la Repubblica e la Porta, secondo quanto affermava il sultano, era messa a dura prova dalla decisione del governo lagunare di impedire agli ottomani di sostenere tramite l'invio di vettovaglie i soldati di stanza a Marano; l'atteggiamento renitente del governo lagunare poteva rischiare di compromettere anche l'alleanza della Serenissima con la Francia, tanto caldeggiata da Solimano. ${ }^{35}$

È probabile che il governo lagunare avesse deciso di bloccare questo traffico di viveri, proprio per non alimentare un clima di paura e di sospetto dei maranesi nei confronti degli ottomani, che già aveva causato disordini e rischiava di compromettere il possesso francese della fortezza.

\subsection{Marano tra l'imperatore Carlo V e l'arciduca Ferdinando}

La reazione arciducale alla "derobatione" di Marano fu sostanzialmente condizionata dal generale quadro politico, ed in particolare dalla guerra coi Turchi in Ungheria. Inizialmente, come raccontò l'ambasciatore Marino Cavalli, «la presa di Marano dolse ed accese sì il re e tutte le persone di quella Corte, che ognuno era malissimo disposto verso questo Eccellentissimo Statom. ${ }^{36}$ Com'era normale, i sospetti di Ferdinando su chi potesse aver ordito il complotto si concentrarono su Venezia, dal momento che non era possibile negare che «la gente che avea tolto Marano non fusse delli subditi» della Repubblica. In ogni caso, continuava l'arciduca, «per onestà, per legge, per capitolazione, quell'Illustrissimo dominio è tenuto, ovvero a restituire e pagare li danni seguiti, ovvero aiutar con tutte le forze la ricuperazione», se una di queste condizioni non venisse esaudita, concludeva, «sarà certissimo argomento che Marano è stato tolto dalla Serenità vostra». ${ }^{37}$

\footnotetext{
${ }^{34}$ Cfr. M. P. Pedani, In nome del Gran Signore. Inviati ottomani a Venezia dalla caduta di Costantinopoli alla guerra di Candia, Venezia, Deputazione Editrice, 1994, p. 152.

${ }^{35}$ ASV, PSCC, b. 214, 2 novembre 1542.

${ }^{36}$ Relazioni di ambasciatori veneti al Senato, Germania (1506-1554) cit., p. 407.

${ }^{37}$ Ibid.
} 
Venezia mise in campo tutta la sua abilità diplomatica per tentare di smarcarsi dalle conseguenze provocate da questo episodio e mantenere, allo stesso tempo, intatta la sua credibilità agli occhi di Ferdinando. In quei giorni concitati, molte missive lasciarono Venezia, dirette soprattutto al luogotenente della Patria, interpellato più volte dagli agenti regi, mandati da Nicolò Della Torre per chiedere ragione di un tale atto lesivo nei confronti della sovranità arciducale.

Estremamente duttile e conciliante è il tono adottato negli scritti destinati all'ambasciatore del re Ferdinando, in cui il Senato sottolineava la sua estraneità ai fatti e il suo desiderio di ripristinare la «quiete et tranquillità di tutta la Christinitade», così brutalmente compromessa. Si trattava di giorni molto delicati per Venezia, che rischiava in ogni momento di veder fallire la fragile tregua con gli imperiali. Ferdinando, nonostante cominciasse a «mitigare l'amaritudine» per lo scacco subito, rimaneva «saldo sul dimandar aiuti per la ricuperazione». Per rafforzare la propria posizione, il Senato si dichiarò pronto a concedere il transito nel suo territorio alle truppe asburgiche che «andarano alla ditta impresa», augurandosi che «Maran ritornerà in mano del Serenissimo Re de Romani», e promettendo di cooperare per sopire i disordini e per punire i facinorosi ancora in libertà. ${ }^{38}$

Venezia si mosse contemporaneamente anche sul piano diplomatico, consigliando al suo ambasciatore Cavalli di distogliere l'attenzione di Ferdinando da Marano. Era necessario che il re dei Romani focalizzasse tutti i suoi sforzi nella difesa dell'Ungheria, minacciata dai Turchi, senza alcuna ulteriore distrazione, sia di uomini che di mezzi, che «se si guadagnava l’Ungheria, Marano cadeva da sé senza difficoltà alcuna». ${ }^{39}$ Oltre a questo, continuò il Cavalli, «niun principe si è veduto da molti anni in qua che abbia voluto tentar ad un tratto due imprese» e Marano "per esser terra, per il sito e per la disperazione di chi v'era dentro» era molto ben difesa. Le scaltre argomentazioni portate dall'ambasciatore veneto furono così efficaci da convincere Ferdinando e, cosa ancor più importante, a proteggere da occhi indiscreti i reconditi progetti che Venezia nutriva sul baluardo maranese.

Venezia, infatti, aveva già intavolato nel settembre del 1542 delle trattative con Francesco de Pazzi, ${ }^{40}$ nominato dallo Strozzi governatore generale di Marano il 3 ottobre 1543. Della cosa vennero tempestivamente avvisati gli ambasciatori veneti alla corte francese, con l'espressa raccomandazione di non farne parole con nessuno. Non era ancora arrivato però il momento della tanto attesa riacquisizione di Marano: per vedere il leone di S. Marco sventolare di nuovo suoi torrioni della

\footnotetext{
${ }^{38}$ ASV, Consiglio dei Dieci, Secreta, R. V, f. 59v.

${ }^{39}$ Relazioni degli ambasciatori veneti al Senato, Germania (1506-1554) cit., pp. 421-422.

${ }^{40}$ ASV, Consiglio dei Dieci, Secreta, R. V, f. 78r, 27 settembre 1542.
} 
fortezza maranese bisognerà aspettare ancora un anno. Il 6 ottobre, infatti, quando Francesco I cedette definitivamente Marano e le sue pertinenze a Pietro Strozzi e ai suoi eredi, a titolo di «dominio dretto et signoria», ${ }^{41}$ la Serenissima si decise ad avviare le negoziazioni, rafforzata nelle sue intenzioni dalla notizia che lo stesso Strozzi, dopo nemmeno un mese, aveva in animo di "prochurar che la terra di Marano et le sue iurisdition pervenissero sotto l'imperio di questo Illustrissimo dominio». L'accordo finale fissò l'acquisizione della fortezza da parte veneziana previo pagamento di 32.500 ducati. Il 26 novembre 1543 il Senato nominò Alessandro Bondumier Provveditore di Marano.

La notizia della "compreda" di Marano giunse alla corte asburgica di Bruxelles a dicembre. L'ambasciatore Bernardo Navagero, di fronte allo sdegno dell'imperatore, tentò di giustificare l'operato del governo lagunare facendo presente che la Repubblica, nonostante fosse stata «molte volte tentata di rihaver Maran, se ben per la perdita di quel loco era stata molte volte in molti travagli, et se ben quel loco è di quella importantia che la prudenzia della maestà vostra può considerar», ${ }^{42}$ non aveva mai accettato o sostenuto nessun progetto di riconquista. In questo caso, però, aveva accettato di trattare con lo Strozzi al solo fine di «schivar il mal nostro, quello della maestà regia et di tutta la christianità» ${ }^{43}$. La mossa del diplomatico veneziano era molto scaltra: puntando l'attenzione sul problema dell'espansionismo ottomano, sperava di guadagnarsi l'approvazione di Carlo V. In realtà l'imperatore si mostrava deluso per non esser stato informato prima di queste trattative, ma anzi averne avuto notizia solo a giochi fatti («io ad un tratto habbia inteso il trattamento et la conclusione»). Carlo V si augurava, però, che «quella Signoria non vorrà tenir quello che è d'altri» ${ }^{44}$, deliberando di restituire la fortezza al suo precedente possessore, il fratello Ferdinando.

I diplomatici veneziani cercarono di conquistarsi la fiducia e l'attenzione dell'imperatore, affinché intercedesse in favore della Repubblica per «acquietar l'animo» del fratello. ${ }^{45}$ Il Consiglio dei Dieci, infatti, aveva consigliato all'ambasciatore Navagero di informare i due sovrani che era ferma intenzione della Repubblica di conservare il possesso di Marano. Da qui i costanti abboccamenti del diplomatico con il principale consigliere dell'imperatore, Nicola Perrenot di Granvelle, «chiamato da Cesare suo primo consigliero e guardia del suo suggello». ${ }^{46} \mathrm{Il}$ Navagero affermava che «le cose ed i negozi di grande importanza non si posso-

\footnotetext{
${ }^{41}$ ASV, PSCC, b. 214, f. 202r, 6 ottobre 1543.

${ }^{42}$ ASV, Archivi propri degli ambasciatori, Germania, b. 1, 23 dicembre 1543.

${ }^{43}$ Ivi.

44 Ivi.

${ }^{45}$ Ivi, 13 marzo 1544.

${ }^{46}$ Relazioni degli ambasciatori veneti al Senato, Germania (1506-1554) cit., p. 503.
} 
no impetrare né condurre a buon fine, senza il particolare favore del Granvela»; questo ministro imperiale godeva di molta influenza presso Carlo $\mathrm{V}$, tanto che quest'ultimo «nelle udienze di persone pubbliche rade volte, anzi mai, si risolve allora, ma rimette a monsignor di Granvela ogni negozio e la risoluzion di esso». ${ }^{47}$ A differenza dei ministri arciducali al servizio di Ferdinando, il Granvelle era ben disposto nei confronti della Serenissima. Anche don Ferrante Gonzaga, governatore generale del Milanese e uomo di fiducia dell'imperatore, si dimostrava disponibile a perorare la causa di Marano presso Carlo V in favore di Venezia («promise di fare con Cesare ogni gagliardo officio»). ${ }^{48}$

Il dilungarsi delle trattative e le sempre maggiori difficoltà accampate dall'arciduca Ferdinando ad accettare l'acquisto veneziano di Marano finirono per preoccupare il governo lagunare: Ferdinando, infatti, permetteva ai suoi ministri di compiere «ogni giorno maggiori et più insopportabili ingiurie nei confronti della popolazione di Marano», alimentando una sempre più diffusa instabilità lungo il confine orientale. ${ }^{49}$

Il nodo della questione riguardava il possesso dei territori esterni alla fortezza maranese: Ferdinando avrebbe anche accettato che la Repubblica mantenesse la fortezza di Marano, ${ }^{50}$ ma solo quella, senza alcun territorio al di fuori delle mura. La risposta veneziana è un netto rifiuto, poiché

niuna città, luogo o castello non s'intende senza il suo territorio, poi che sendo le possessioni et l'entrate di quelli del loco di Marano sparse in tutto il territorio, che l'haver vostra Serenità la Terra et parte del territorio, et il serenissimo re il resto, era un dar occasione alli sudditi dell'una et l'altra di star in continue risse, si come suole esser sempre nelli luoghi che hanno le giurisdittioni vicine et confuse, dal che ne potria succeder qualche maggior scandalo. ${ }^{51}$

È probabile che in seguito, dal momento che nei documenti successivi non viene più menzionata la questione, il governo lagunare si fosse risolto ad accettare (controvoglia) l'offerta di Ferdinando. Nonostante il raggiungimento di un accordo,

\footnotetext{
${ }^{47}$ Ivi, pp. 499 e 504.

${ }^{48}$ ASV, Archivi propri degli ambasciatori, Germania, b. 1, 15 marzo 1544.

${ }^{49}$ Ivi, 3 giugno 1544.

${ }^{50}$ Già nel marzo del 1544, circolavano voci presso la corte di Vienna che Ferdinando non pretendesse più la restituzione di Marano, anzi che sarebbe stato disposto a «componer la cosa in qualche somma de dinari, o darli qualche altro loco in cambio». Se la Repubblica avesse sin da subito resa nota la sua intenzione di acquistare la fortezza, secondo l'arciduca l'avrebbe ottenuta facilmente con la sua approvazione, nonostante i suoi ministri affermassero che «lassarlo hora vada della dignità di sua maestà» (alimentando ulteriormente l'ostilità dell'arciduca nei confronti di Venezia); Ivi, 27 marzo 1544.
}

${ }^{51}$ Ivi, 7 giugno 1545. 
però, anche se non del tutto favorevole alla Repubblica di Venezia, fra le due parti continuò a permanere un clima di ostilità latente. Ancora nel 1548, l'ambasciatore Lorenzo Contarini constatava che lo sdegno di Ferdinando per la vicenda di Marano non si era placato (su Marano «mai non ragiona che non si senta mutar tutta, e non vuol sentire alcuna ragione in difesa»). Il risentimento del re dei Romani era del resto fomentato dai suoi cortigiani e ministri. Il diplomatico veneziano era però convinto che Ferdinando non si sarebbe mai mosso senza l'appoggio del fratello e che, d'altra parte, i due Asburgo sarebbero stati trattenuti dai ben più urgenti problemi che dovevano affrontare in tutta Europa. Perciò, concludeva, «alla cosa di Marano non vedo altro rimedio che lasciar correre il tempo», poiché solo il tempo poteva legittimare le usurpazioni. ${ }^{52}$

\section{Conclusioni}

La presa di Marano (1542) compiuta dal Sacchia, da piccola scaramuccia di confine, finì per tramutarsi in un vero e proprio affaire diplomatico, catapultando la piccola fortezza al centro degli interessi politici delle maggiori potenze dell'epoca, punto focale all'interno del grande conflitto europeo che contrapponeva Carlo V e il fratello a Francesco I, alleato di Solimano il Magnifico. Rispetto a questa contesa, Venezia mantenne un atteggiamento neutrale, salvo poi portare a termine una “negoziazione-lampo" con Pietro Strozzi nel 1543, perciò sottraendo la fortezza all'arciduca Ferdinando d'Asburgo: questo contribuì in maniera determinante ad inasprire e a rafforzare il clima di ostilità latente che già caratterizzava i rapporti confinari veneto-arciducali. Dopo la crisi di Marano, lo stato delle difese della Patria del Friuli continuò a suscitare una preoccupazione perenne presso gli organi del governo lagunare che, attraverso la progettazione di Palmanova sul finire del secolo (1593), tentarono di dare una soluzione definitiva ai cronici problemi di politica confinaria con gli arciducali.

\footnotetext{
${ }^{52}$ Relazioni degli ambasciatori veneti al Senato, Germania (1506-1554) cit., Pp. 800-802. Nel 1548 una lettera di un certo fra Tulio Morato di Capodistria avvertiva i Capi del Consiglio dei Dieci che alcuni di Marano meditavano di tradire i veneziani «dacuordo con quelli di Maraneto, dove si aspetta vegni la sua volta di operare tal cosa et introdure dentro quelli di fuora", in questo affare erano coinvolti anche alcuni castellani tedeschi; in ASV, Capi Consiglio dei Dieci, Lettere rettori, b. 256, f. 273, 27 ottobre 1548.
} 